\title{
Some Hermite-Hadamard type integral inequalities for operator AG-preinvex functions
}

\author{
Ali Taghavi \\ Department of Mathematics, \\ Faculty of Mathematical Sciences, \\ University of Mazandaran, Iran \\ email: taghavi@umz.ac.ir
}

\section{Haji Mohammad Nazari}

Department of Mathematics, Faculty of Mathematical Sciences, University of Mazandaran, Iran email: m.nazari@stu.umz.ac.ir

\author{
Vahid Darvish \\ Department of Mathematics, \\ Faculty of Mathematical Sciences, \\ University of Mazandaran, Iran \\ email: vahid.darvish@mail.com \\ (vdarvish@wordpress.com)
}

\begin{abstract}
In this paper, we introduce the concept of operator AGpreinvex functions and prove some Hermite-Hadamard type inequalities for these functions. As application, we obtain some unitarily invariant norm inequalities for operators.
\end{abstract}

\section{Introduction and preliminaries}

The following Hermite-Hadamard inequality holds for any convex function $f$ defined on $\mathbb{R}$

$$
(b-a) f\left(\frac{a+b}{2}\right) \leq \int_{a}^{b} f(x) d x \leq(b-a) \frac{f(a)+f(b)}{2}, \quad a, b \in \mathbb{R} .
$$

2010 Mathematics Subject Classification: 47A63, 15A60, 47B05, 47B10, 26D15

Key words and phrases: Hermite-Hadamard inequality, operator AG-preinvex function, log-convex function, positive linear operator 
It was firstly discovered by Hermite in 1881 in the journal Mathesis (see [8]). But this result was nowhere mentioned in the mathematical literature and was not widely known as Hermite's result [10].

Beckenbach, a leading expert on the history and the theory of convex functions, wrote that this inequality was proven by Hadamard in 1893 [2]. In 1974, Mitrinovič found Hermites note in Mathesis [8]. Since (1) was known as Hadamards inequality, the inequality is now commonly referred as the Hermite-Hadamard inequality [10].

Definition 1 [13] A continuous function $\mathrm{f}: \mathrm{I} \subset \mathbb{R} \rightarrow \mathbb{R}^{+}$is said to be an AG-convex function (arithmetic-geometrically or log-convex function) on the interval I if

$$
f(\lambda a+(1-\lambda) b) \leq f(a)^{\lambda} f(b)^{1-\lambda} .
$$

for $\mathrm{a}, \mathrm{b} \in \mathrm{I}$ and $\lambda \in[0,1]$, i.e., $\log \mathrm{f}$ is convex.

Theorem 1 [13] Let $\mathrm{f}$ be an AG-convex function defined on $[\mathrm{a}, \mathrm{b}]$. Then, we have

$$
\begin{aligned}
f\left(\frac{a+b}{2}\right) & \leq \sqrt{f\left(\frac{3 a+b}{4}\right) f\left(\frac{a+3 b}{4}\right)} \\
& \leq \exp \left(\frac{1}{b-a} \int_{a}^{b} \log (f(u)) d u\right) \\
& \leq \sqrt{f\left(\frac{a+b}{2}\right)} \cdot \sqrt[4]{f(a)} \cdot \sqrt[4]{f(b)} \\
& \leq \sqrt{f(a) f(b)}
\end{aligned}
$$

where $\mathbf{u}=\log \mathbf{t}$.

Let $\mathrm{B}(\mathrm{H})$ stands for the $\mathrm{C}^{*}$-algebra of all bounded linear operators on a complex Hilbert space $H$ with inner product $\langle\cdot, \cdot\rangle$. An operator $A \in B(H)$ is positive and write $A \geq 0$ if $\langle A x, x\rangle \geq 0$ for all $x \in H$. Let $B(H)_{\text {sa }}$ stand for the set of all self-adjoint elements of $B(H)$.

Let $A$ be a self-adjoint operator in $B(H)$. The Gelfand map establishes a *-isometrically isomorphism $\Phi$ between the set $\mathrm{C}(\operatorname{Sp}(A))$ of all continuous functions defined on the spectrum of $A$, denoted by $\operatorname{Sp}(A)$, and the $C^{*}$-algebra $C^{*}(A)$ generated by $A$ and the identity operator $1_{H}$ on $H$ as follows:

for any $f, g \in C(\operatorname{Sp}(A)))$ and any $\alpha, \beta \in \mathbb{C}$ we have:

- $\Phi(\alpha f+\beta g)=\alpha \Phi(f)+\beta \Phi(g)$; 
- $\Phi(f g)=\Phi(f) \Phi(g)$ and $\Phi(\bar{f})=\Phi(f)^{*}$;

- $\|\Phi(f)\|=\|f\|:=\sup _{t \in \operatorname{Sp}(A)}|\mathbf{f}(\mathbf{t})|$;

- $\Phi\left(f_{0}\right)=1_{H}$ and $\Phi\left(f_{1}\right)=A$, where $f_{0}(t)=1$ and $f_{1}(t)=t$, for $t \in \operatorname{Sp}(A)$.

With this notation we define

$$
f(A)=\Phi(f) \text { for all } f \in C(\operatorname{Sp}(A))
$$

and we call it the continuous functional calculus for a self-adjoint operator $A$.

If $A$ is a self-adjoint operator and $f$ is a real valued continuous function on $\operatorname{Sp}(A)$, then $f(t) \geq 0$ for any $t \in \operatorname{Sp}(A)$ implies that $f(A) \geq 0$, i.e. $f(A)$ is a positive operator on $H$. Moreover, if both $f$ and $g$ are real valued functions on $\operatorname{Sp}(A)$ then the following important property holds:

$$
f(t) \geq g(t) \text { for any } t \in \operatorname{Sp}(A) \text { implies that } f(A) \geq g(A) \text {, }
$$

in the operator order of $\mathrm{B}(\mathrm{H})$, see [14].

Definition 2 A real valued continuous function $\mathrm{f}$ on an interval $\mathrm{I}$ is said to be operator convex function if

$$
f(\lambda A+(1-\lambda) B) \leq \lambda f(A)+(1-\lambda) f(B),
$$

in the operator order, for all $\lambda \in[0,1]$ and self-adjoint operators $\mathrm{A}$ and $\mathrm{B}$ in $\mathrm{B}(\mathrm{H})$ whose spectra are contained in $\mathrm{I}$.

In [4] Dragomir investigated the operator version of the Hermite-Hadamard inequality for operator convex functions. Let $f: I \rightarrow \mathbb{R}$ be an operator convex function on the interval I then, for any self-adjoint operators $A$ and $B$ with spectra in I, the following inequalities holds

$$
\begin{aligned}
f\left(\frac{A+B}{2}\right) & \leq 2 \int_{\frac{1}{4}}^{\frac{3}{4}} f(t A+(1-t) B) d t \\
& \leq \frac{1}{2}\left[f\left(\frac{3 A+B}{4}\right)+f\left(\frac{A+3 B}{4}\right)\right] \\
& \leq \int_{0}^{1} f((1-t) A+t B) d t \\
& \leq \frac{1}{2}\left[f\left(\frac{A+B}{2}\right)+\frac{f(A)+f(B)}{2}\right] \\
& \leq \frac{f(A)+f(B)}{2},
\end{aligned}
$$


for the first inequality in above, see [12].

In [5], Ghazanfari et al. gave the concept of operator preinvex function and obtained Hermite-Hadamard type inequality for operator preinvex function.

Definition 3 [5] Let $\mathrm{X}$ be a real vector space, a set $\mathrm{S} \subseteq \mathrm{X}$ is said to be invex with respect to the map $\eta: S \times S \rightarrow X$, if for every $x, y \in S$ and $t \in[0,1]$,

$$
x+\operatorname{t\eta }(x, y) \in S \text {. }
$$

It is obvious that every convex set is invex with respect to the map $\eta(x, y)=$ $x-y$, but there exist invex sets which are not convex (see [1]).

Let $S \subseteq X$ be an invex set with respect to $\eta$. For every $x, y \in S$. the $\eta$-path $P_{x v}$ joining the points $x$ and $v:=x+\eta(y, x)$ is defined as follows

$$
P_{x v}:=\{z: z=x+\operatorname{t\eta }(y, x), t \in[0,1]\} .
$$

The mapping $\eta$ is said to satisfy the condition (C) if for every $x, y \in S$ and $\mathrm{t} \in[0,1]$,

$$
\eta(y, y+\operatorname{t\eta }(y, x))=-\operatorname{t\eta }(x, y), \quad \eta(x, y+\operatorname{t\eta }(x, y))=(1-t) \eta(x, y) .
$$

Note that for every $x, y \in S$ and every $t_{1}, t_{2} \in[0,1]$, from conditions in (C), we have

$$
\eta\left(y+t_{2} \eta(x, y), y+t_{1} \eta(x, y)\right)=\left(t_{2}-t_{1}\right) \eta(x, y),
$$

see [9] for details.

Definition 4 Let $\mathrm{S} \subseteq \mathrm{B}(\mathrm{H})_{\text {sa }}$ be an invex set with respect to $\eta: \mathrm{S} \times \mathrm{S} \rightarrow$ $\mathrm{B}(\mathrm{H})_{\text {sa. }}$. Then, the continuous $\mathrm{f}: \mathbb{R} \rightarrow \mathbb{R}$ is said to be operator preinvex with respect to $\eta$ on $S$, if for every $A, B \in S$ and $\mathrm{t} \in[0,1]$,

$$
f(A+\operatorname{t\eta }(B, A)) \leq(1-t) f(A)+t f(B),
$$

in the operator order in $\mathrm{B}(\mathrm{H})$.

Every operator convex function is operator preinvex with respect to the map $\eta(A, B)=A-B$, but the converse does not hold (see [5]).

Theorem 2 [5] Let $\mathrm{S} \subseteq \mathrm{B}(\mathrm{H})_{\text {sa }}$ be an invex set with respect to $\eta: \mathrm{S} \times \mathrm{S} \rightarrow$ $\mathrm{B}(\mathrm{H})_{\text {sa }}$ and $\eta$ satisfies condition $(\mathrm{C})$. If for every $\mathrm{A}, \mathrm{B} \in \mathrm{S}$ and $\mathrm{V}=\mathrm{A}+\eta(\mathrm{B}, \mathrm{A})$ the function $\mathrm{f}: \mathrm{I} \subseteq \mathbb{R} \rightarrow \mathbb{R}$ is operator preinvex with respect to $\eta$ on $\eta$-path $\mathrm{P}_{\mathrm{A} V}$ with spectra of $\mathrm{A}$ and spectra of $\mathrm{V}$ in the interval $\mathrm{I}$. Then we have the inequality

$$
f\left(\frac{A+V}{2}\right) \leq \int_{0}^{1} f\left((A+\operatorname{t\eta }(B, A)) d t \leq \frac{f(A)+f(B))}{2} .\right.
$$


Throughout this paper, we introduce the concept of operator AG-preinvex functions and obtain some Hermite-Hadamard type inequalities for these class of functions. These results lead us to obtain some inequalities unitarily invariant norm inequalities for operators.

\section{Some inequalities for operator AG-preinvex func- tions}

In this section, we prove some Hermite-Hadamard type inequalities for operator AG-preinvex functions.

Definition 5 [13] A continuous function $\mathrm{f}: \mathrm{I} \subseteq \mathbb{R} \rightarrow \mathbb{R}^{+}$is said to be operator $A G$-convex (concave) if

$$
f(\lambda A+(1-\lambda) B) \leq(\geq) f(A)^{\lambda} f(B)^{1-\lambda}
$$

for $0 \leq \lambda \leq 1$ and self-adjoint operators $\mathrm{A}$ and $\mathrm{B}$ in $\mathrm{B}(\mathrm{H})$ whose spectra are contained in $\mathrm{I}$.

Example 1 [6, Corollary 7.6.8] Let $\mathrm{A}$ and $\mathrm{B}$ be to positive definite $\mathrm{n} \times \mathrm{n}$ complex matrices. For $0<\alpha<1$, we have

$$
|\alpha A+(1-\alpha) B| \geq|A|^{\alpha}|B|^{1-\alpha}
$$

where $|\cdot|$ denotes determinant of a matrix.

Let $f$ be an operator AG-convex function, for commutative positive operators $A, B \in B(H)$ whose spectra are contained in $I$, then we have

$$
\begin{aligned}
f\left(\frac{A+B}{2}\right) & \leq \int_{0}^{1} \sqrt{f(\alpha A+(1-\alpha) B) f((1-\alpha) A+\alpha B)} d \alpha \\
& \leq \sqrt{f(A) f(B)},
\end{aligned}
$$

(see [13] for more inequalities).

Definition 6 Let $\mathrm{S} \subseteq \mathrm{B}(\mathrm{H})_{\text {sa }}$ be an invex set with respect to $\eta: \mathrm{S} \times \mathrm{S} \rightarrow$ $\mathrm{B}(\mathrm{H})_{\text {sa. }}$. A continuous function $\mathrm{f}: \mathrm{I} \subseteq \mathbb{R} \rightarrow \mathbb{R}^{+}$is called operator $A G$-preinvex with respect to $\eta$ on $\mathrm{S}$ if

$$
f(A+\operatorname{t\eta }(B, A)) \leq f(A)^{1-t} f(B)^{t}
$$

for $\mathrm{t} \in[0,1]$ such that spectra of $\mathrm{A}$ and $\mathrm{B}$ are contained in $\mathrm{I}$. 
Remark 1 Let $\mathrm{f}$ be an operator AG-preinvex function, in a commutative case, we then get

$$
\begin{aligned}
f(A+\operatorname{t\eta }(B, A)) & \leq f(A)^{1-t} f(B)^{t} \\
& \leq(1-t) f(A)+\operatorname{tf}(B) \\
& \leq \max \{f(A), f(B)\}
\end{aligned}
$$

It means that $\mathrm{f}$ is operator quasi preinvex i.e., $\mathrm{f}(\mathrm{A}+\mathrm{t} \eta(\mathrm{B}, \mathrm{A})) \leq \max \{\mathrm{f}(\mathrm{A}), \mathrm{f}(\mathrm{B})\}$.

We need the following lemma for giving Hermite-Hadamard type inequalities for operator preinvex function.

Lemma 1 Let $\mathrm{S} \subseteq \mathrm{B}(\mathrm{H})_{\text {sa }}$ be an invex set with respect to $\eta: \mathrm{S} \times \mathrm{S} \rightarrow \mathrm{B}(\mathrm{H})_{\text {sa }}$ and $\mathrm{f}: \mathrm{I} \subseteq \mathbb{R} \rightarrow \mathbb{R}^{+}$be a continuous function on the interval $\mathrm{I}$. Suppose that $\eta$ satisfies condition $(C)$. Then for every $A, B \in S$ and $V=A+\eta(B, A)$ and for some fixed $s \in(0,1]$ the function $\mathrm{f}$ is operator $A G$-preinvex with respect to $\eta$ on $\eta$-path $\mathrm{P}_{\mathrm{AV}}$ with spectra of $\mathrm{A}$ and $\mathrm{V}$ in the interval $\mathrm{I}$ if and only if the function $\varphi_{\mathrm{A}, \mathrm{B}}$ defined by

$$
\varphi_{A, B}(t)=f(A+\operatorname{t\eta }(B, A))
$$

is a log-convex function on $[0,1]$.

Proof. Let $\varphi$ be a log-convex function on $[0,1]$, we should prove that $f$ is operator AG-preinvex with respect to $\eta$.

For every $C_{1}:=A+t_{1} \eta(B, A) \in P_{A V}, C_{2}:=A+t_{2} \eta(B, A) \in P_{A V}$, fixed $\lambda \in[0,1]$, by (9) we have

$$
\begin{aligned}
f\left(C_{1}+\lambda \eta\left(C_{2}, C_{1}\right)\right) & =f\left(A+t_{1} \eta(B, A)+\lambda \eta\left(A+t_{2} \eta(B, A), A+t_{1} \eta(B, A)\right)\right) \\
& =f\left(A+t_{1} \eta(B, A)+\lambda\left(t_{2}-t_{1}\right) \eta(B, A)\right) \\
& =f\left(A+\left(t_{1}+\lambda t_{2}-\lambda t_{1}\right) \eta(B, A)\right) \\
& =f\left(A+\left((1-\lambda) t_{1}+\lambda t_{2}\right) \eta(B, A)\right) \\
& =\varphi\left((1-\lambda) t_{1}+\lambda t_{2}\right) \\
& \leq \varphi\left(t_{1}\right)^{1-\lambda} \varphi\left(t_{2}\right)^{\lambda} \\
& =\left(f\left(A+t_{1} \eta(B, A)\right)\right)^{1-\lambda}\left(f\left(A+t_{2} \eta(B, A)\right)\right)^{\lambda} .
\end{aligned}
$$


Conversely, let $f$ be operator AG-preinvex, then, by (6)

$$
\begin{aligned}
\varphi\left((1-\lambda) t_{1}+\lambda t_{2}\right) & =f\left(A+\left((1-\lambda) t_{1}+\lambda t_{2}\right) \eta(B, A)\right) \\
& =f\left(A+t_{1} \eta(B, A)+\lambda\left(t_{2}-t_{1}\right) \eta(B, A)\right) \\
& =f\left(A+t_{1} \eta(B, A)+\lambda \eta\left(A+t_{2} \eta(B, A), A+t_{1} \eta(B, A)\right)\right) \\
& \leq f\left(A+t_{1} \eta(B, A)\right)^{1-\lambda} f\left(A+t_{2} \eta(B, A)\right)^{\lambda} \\
& =\varphi\left(t_{1}\right)^{1-\lambda} \varphi\left(t_{2}\right)^{\lambda} .
\end{aligned}
$$

Theorem 3 Let $\mathrm{S} \subseteq \mathrm{B}(\mathrm{H})_{\text {sa }}$ be an invex set with respect to $\eta: \mathrm{S} \times \mathrm{S} \rightarrow \mathrm{B}(\mathrm{H})_{\text {sa }}$ and $\mathrm{f}: \mathrm{I} \subseteq \mathbb{R} \rightarrow \mathbb{R}^{+}$be a continuous function on the interval I. Suppose that $\eta$ satisfies condition $(C)$. Then for the operator $A G$-preinvex function $\mathrm{f}$ with respect to $\eta$ on $\eta$-path $\mathrm{P}_{\mathrm{AV}}$ such that spectra of $\mathrm{A}$ and $\mathrm{V}$ are in $\mathrm{I}$, we have

$$
\begin{aligned}
f\left(\frac{A+V}{2}\right) & \leq \sqrt{f\left(\frac{3 A+V}{4}\right) f\left(\frac{A+3 V}{4}\right)} \\
& \leq \exp \left(\int_{0}^{1} \log (f(A+\operatorname{tn}(B, A))) d t\right) \\
& \leq \sqrt{f\left(\frac{A+V}{2}\right) \sqrt[4]{f(A)} \sqrt[4]{f(V)}} \\
& \leq \sqrt{f(A) f(V)} \\
& \leq \frac{f(A)+f(V)}{2}
\end{aligned}
$$

where $\mathrm{A}, \mathrm{B} \in \mathrm{S}$ and $\mathrm{V}=\mathrm{A}+\eta(\mathrm{B}, \mathrm{A})$ and for some fixed $\mathrm{s} \in(0,1]$

Proof. Since $f$ is an operator AG-preinvex function, so by Lemma 1 we have $\varphi(t)=f(A+\operatorname{t\eta }(B, A))$ is log-convex on $[0,1]$.

On the other hand, in [11] we obtained the following inequalities for logconvex function $\varphi$ on $[0,1]$ :

$$
\begin{aligned}
\varphi\left(\frac{1}{2}\right) & \leq \sqrt{\varphi\left(\frac{1}{4}\right) \varphi\left(\frac{3}{4}\right)} \\
& \leq \exp \left(\int_{0}^{1} \log (\varphi(u)) d u\right)
\end{aligned}
$$




$$
\begin{aligned}
& \leq \sqrt{\varphi\left(\frac{1}{2}\right)} \cdot \sqrt[4]{\varphi(0)} \cdot \sqrt[4]{\varphi(1)} \\
& \leq \sqrt{\varphi(0) \varphi(1)}
\end{aligned}
$$

By knowing that

$$
\begin{aligned}
\varphi(0) & =f(A) \\
\varphi\left(\frac{1}{4}\right) & =f\left(A+\frac{1}{4} \eta(B, A)\right)=f\left(\frac{3 A+V}{4}\right) \\
\varphi\left(\frac{1}{2}\right) & =f\left(A+\frac{1}{2} \eta(B, A)\right)=f\left(\frac{A+V}{2}\right) \\
\varphi(1) & =f(V),
\end{aligned}
$$

we obtain

$$
\begin{aligned}
f\left(\frac{A+V}{2}\right) & \leq \sqrt{f\left(\frac{3 A+V}{4}\right) f\left(\frac{A+3 V}{4}\right)} \\
& \leq \exp \left(\int_{0}^{1} \log (f(A+\operatorname{tn}(B, A)) d t)\right. \\
& \leq \sqrt{f\left(\frac{A+V}{2}\right)} \sqrt[4]{f(A)} \sqrt[4]{f(V)} \\
& \leq \sqrt{f(A) f(V)} .
\end{aligned}
$$

\section{Some unitarily invariant norm inequalities for op- erator AG-preinvex functions}

In this section we prove some unitarily invariant norm inequalities for operators.

We consider the wide class of unitarily invariant norms $|\|\cdot \mid\|$. Each of these norms is defined on an ideal in $\mathrm{B}(\mathrm{H})$ and it will be implicitly understood that when we talk of $\||T|\|$, then the operator $T$ belongs to the norm ideal associated with $|\|\cdot \mid\|$. Each unitarily invariant norm $|\|\cdot \mid\|$ is characterized by the invariance property $\|\mathrm{UTV}\|\|=\| \mathrm{IT} \|$ for all operators $\mathrm{T}$ in the norm ideal associated with $|\|\cdot \mid\|$ and for all unitary operators $U$ and $V$ in $B(H)$. 
For $1 \leq p<\infty$, the Schatten $p$-norm of a compact operator $A$ is defined by $\|A\|_{p}=\left(\operatorname{Tr}|A|^{p}\right)^{1 / p}$, where $\operatorname{Tr}$ is the usual trace functional. Note that for compact operator $A$ we have, $\|A\|=s_{1}(A)$, and if $A$ is a Hilbert-Schmidt operator, then $\|A\|_{2}=\left(\sum_{j=1}^{\infty} s_{j}^{2}(A)\right)^{1 / 2}$. These norms are special examples of the more general class of the Schatten $p$-norms which are unitarily invariant [3].

Remark 2 The author of [7] proved that if $\mathrm{A}, \mathrm{B}, \mathrm{X} \in \mathrm{B}(\mathrm{H})$ such that $\mathrm{A}, \mathrm{B}$ are positive operators, then for $0 \leq v \leq 1$ we have

$$
\left\|A^{v} X B^{1-v}|\|\leq\| A X|\right\|^{v} \| X B||^{1-v} .
$$

Let $\mathrm{X}=\mathrm{I}$ in above inequality, we then get

$$
\left\|A^{v} B^{1-v}\right\|\left|\leq\left\|A\left|\left\|^{v}\right\|\right| B\right\|^{1-v} .\right.
$$

Lemma 2 Let $\mathrm{f}$ be an operator AG-preinvex function and $\eta$ satisfies the condition $(C)$. Then the function $\varphi_{\mathrm{A}, \mathrm{B}}:[0,1] \rightarrow \mathbb{R}$ defined as follows

$$
\varphi(t)=\|f(A+\operatorname{t\eta }(B, A)) \mid\|
$$

is log-convex.

Proof. Let $t_{1}, t_{2} \in[0,1]$, we have

$$
\begin{aligned}
\varphi\left((1-\lambda) t_{1}+\lambda t_{2}\right) & =\left\|f\left(A+\left((1-\lambda) t_{1}+\lambda t_{2}\right) \eta(B, A)\right)\right\| \\
& =\left\|f\left(A+t_{1} \eta(B, A)+\lambda\left(t_{2}-t_{1}\right) \eta(B, A)\right)\right\| \\
& =\left\|f\left(A+t_{1} \eta(B, A)+\lambda \eta\left(A+t_{2} \eta(B, A), A+t_{1} \eta(B, A)\right)\right)\right\| \\
& \leq\left\|f\left(A+t_{1} \eta(B, A)\right)^{1-\lambda} f\left(A+t_{2} \eta(B, A)\right)^{\lambda}\right\| \| \\
& \leq\left.\left\|f\left(A+t_{1} \eta(B, A)\right)\right\|\right|^{1-\lambda}\left\|f\left(A+t_{2} \eta(B, A)\right)\right\|^{\lambda} \text { by }(12) \\
& =\varphi\left(t_{1}\right)^{1-\lambda} \varphi\left(t_{2}\right)^{\lambda} .
\end{aligned}
$$

Theorem 4 Let $\mathrm{S} \subseteq \mathrm{B}(\mathrm{H})_{\text {sa }}$ be an invex set with respect to $\eta: \mathrm{S} \times \mathrm{S} \rightarrow \mathrm{B}(\mathrm{H})_{\text {sa }}$ and $\mathrm{f}: \mathrm{I} \subseteq \mathbb{R} \rightarrow \mathbb{R}^{+}$be a continuous function on the interval $\mathrm{I}$. Suppose that $\eta$ satisfies condition $(C)$. Then for the operator AG-preinvex function $\mathrm{f}$ with 
respect to $\eta$ on $\eta$-path $\mathrm{P}_{\mathrm{AV}}$ such that spectra of $\mathrm{A}$ and $\mathrm{V}$ are in $\mathrm{I}$, we have

$$
\begin{aligned}
\left\|f\left(\frac{A+V}{2}\right)\right\| \| & \leq \sqrt{\left\|f\left(\frac{3 A+V}{4}\right)\right\|\|\| f\left(\frac{A+3 V}{4}\right)\|\|} \\
& \leq \exp \left(\int_{0}^{1} \log (\|f(A+\operatorname{t\eta }(B, A))\| \|) d t\right) \\
& \leq \sqrt{\left\|f\left(\frac{A+V}{2}\right)\right\|} \sqrt[4]{\|f(A)\| \sqrt[4]{\|f(V)\|}} \\
& \leq \sqrt{\|f(A)\|\|\| f(V) \|} \\
& \leq \frac{\|f(A)\|\|+\| f(V) \|}{2} .
\end{aligned}
$$

where $A, B \in S$ and $V=A+\eta(B, A)$ and for some fixed $s \in(0,1]$

Proof. Since $f$ is an operator AG-preinvex function, so by Lemma 2 we have $\varphi(t)=\|f(A+\operatorname{t\eta }(B, A)) \mid\|$ is log-convex on $[0,1]$.

On the other hand, in [11] we obtained the following inequalities for logconvex function $\varphi$ on $[0,1]$ :

$$
\begin{aligned}
\varphi\left(\frac{1}{2}\right) & \leq \sqrt{\varphi\left(\frac{1}{4}\right) \varphi\left(\frac{3}{4}\right)} \\
& \leq \exp \left(\int_{0}^{1} \log (\varphi(u)) d u\right) \\
& \leq \sqrt{\varphi\left(\frac{1}{2}\right)} \cdot \sqrt[4]{\varphi(0)} \cdot \sqrt[4]{\varphi(1)} \\
& \leq \sqrt{\varphi(0) \varphi(1)} .
\end{aligned}
$$

By knowing that

$$
\begin{aligned}
\varphi(0) & =\|f(A)\| \\
\varphi\left(\frac{1}{4}\right) & =\left\|f\left(A+\frac{1}{4} \eta(B, A)\right)\right\|=\left\|f\left(\frac{3 A+V}{4}\right)\right\| \| \\
\varphi\left(\frac{1}{2}\right) & =\left\|f\left(A+\frac{1}{2} \eta(B, A)\right)\right\|=\left\|f\left(\frac{A+V}{2}\right)\right\| \| \\
\varphi(1) & =\|f(V)\|,
\end{aligned}
$$


we obtain

$$
\begin{aligned}
\left\|f\left(\frac{A+V}{2}\right)\right\| \| & \leq \sqrt{\left\|f\left(\frac{3 A+V}{4}\right)\right\|\|\| f\left(\frac{A+3 V}{4}\right)\|\|} \\
& \leq \exp \left(\int_{0}^{1} \log (\|f(A+\operatorname{t\eta }(B, A))\| \|) d t\right) \\
& \leq \sqrt{\|\| f\left(\frac{A+V}{2}\right)\|\|} \sqrt[4]{\|f(A)\| \sqrt[4]{\|f(V)\| \|}} \\
& \leq \sqrt{\|f(A)\|\|\| f(V) \|} .
\end{aligned}
$$

Let $\eta(B, A)=B-A$ in the above theorem, then we obtain the following inequalities:

$$
\begin{aligned}
\left\|f\left(\frac{A+B}{2}\right)\right\| \| & \leq \sqrt{\left\|f\left(\frac{3 A+B}{4}\right)\right\|\|\| f\left(\frac{A+3 B}{4}\right)\|\|} \\
& \leq \exp \left(\int_{0}^{1} \log (\|f((1-t) A+t B)\| d t)\right. \\
& \leq \sqrt{\left\|f\left(\frac{A+B}{2}\right)\right\|} \sqrt[4]{\|f(A)\| \sqrt[4]{\|f(B)\| \mid}} \\
& \leq \sqrt{\||f(A)|\| \mid\|f(B)\|} \\
& \leq \frac{\|f(A)\||+|\|f(B)\|}{2} .
\end{aligned}
$$

\section{References}

[1] T. Antczak, Mean value in invexity analysis, Nonlinear Anal., 60 (2005), $1473-1484$

[2] E. F. Beckenbach, Convex functions, Bull. Amer. Math. Soc., 54 (1948), 439-460.

[3] R. Bhatia, Matrix Analysis, GTM 169, Springer-Verlag, New York, 1997.

[4] S. S. Dragomir, Hermite-Hadamards type inequalities for operator convex functions, Applied Mathematics and Computation., 218 (2011), 766-772. 
[5] A. G. Ghazanfari, M. Shakoori, A. Barani, S. S. Dragomir, Hermite-Hadamard type inequality for operator preinvex functions, arXiv:1306.0730v1

[6] R. A. Horn, C. R. Johnson, Matrix Analysis, Cambridge University Press, 2012.

[7] F. Kittaneh, Norm inequalities for fractional powers of positive operators, Lett. Math. Phys., 27 (1993), 279-285.

[8] D. S. Mitrinović, I. B. Lacković, Hermite and convexity, Aequationes Math., 28 (1985), 229-232.

[9] S. R. Mohan, S. K. Neogy, On invex sets and preinvex function, J. Math. Anal. Appl., 189 (1995), 901-908.

[10] J. E. Pečarić, F. Proschan, Y. L. Tong, Convex Functions, Partial Orderings, and Statistical Applications, Academic Press Inc., San Diego, 1992.

[11] A. Taghavi, V. Darvish, H. M. Nazari, S. S. Dragomir, Hermite-Hadamard type inequalities for operator geometrically convex functions, Monatsh. Math. 10.1007/s00605-015-0816-6.

[12] A. Taghavi, V. Darvish, H. M. Nazari, S. S. Dragomir, Some inequalities associated with the Hermite-Hadamard inequalities for operator $\mathrm{h}$ convex functions, Accepted for publishing by J. Adv. Res. Pure Math., (http://rgmia.org/papers/v18/v18a22.pdf)

[13] A. Taghavi, V. Darvish, H. M. Nazari, Some integral inequalities for operator arithmetic-geometrically convex functions, arXiv:1511.06587v1

[14] K. Zhu, An introduction to operator algebras, CRC Press, 1993. 